explain differences in all ages and premature CVD mortality between LAs in England.

Methods All data were sourced for each LA in England. Outcome variables were age-standardised 2012 to 2014 CVD mortality for all ages and those under 75 (premature mortality). Prevalence of ethnic and socioeconomic groups from the UK 2011 census, Public Health England data on index of multiple deprivation (IMD) score, prevalence of smoking, physical activity and obesity/overweight and Ordnance Survey environmental data on percentage of food shops, eating out shops, green/blue space, sporting facilities and health facilities were sourced. We used the Akaike Information Criterion (AIC) to assess which types of variables provided the best statistical model to explain variation in CVD mortality between LAs then used multiple linear regression to assess which variables remained associated with the outcome.

Results Including health, demographic, environment and IMD variables provided the best fit for explaining variation in CVD mortality at all ages, with an adjusted R2 of 0.63 . For premature CVD mortality, excluding environmental data improved the fit of the model and gave an adjusted R2 of 0.82 .

The percentage of Indian and Pakistani ethnic groups in LAs remained associated with all ages CVD mortality, along with higher scores for the employment domain and living environment domain of the IMD. For premature mortality, the percentage of Pakistani and Bangladeshi ethnic groups, excess weight prevalence and higher income and crime IMD scores remained associated.

Conclusion Certain IMD domains and prevalence of some South Asian ethnic groups are important for explaining variation in age-standardised cardiovascular disease mortality at the LA level in England. These findings are valuable for understanding which factors to target to reduce inequalities in CVD mortality between LAs in England.

\section{P35 ARE PATIENT OUTCOMES IMPROVING? MAJOR AMPUTATION AND DEATH FOLLOWING LOWER LIMB REVASCULARISATION PROCEDURES IN ENGLAND}

\begin{abstract}
1,2K Heikkila*, ${ }^{3} \mathrm{DC}$ Mitchell, ${ }^{4} \mathrm{IM}$ Loftus, ${ }^{1,2} \mathrm{DA}$ Cromwell. ${ }^{1}$ Health Services Research and Policy, London School of Hygiene and Tropical Medicine, London, UK; ${ }^{2}$ Clinical Effectiveness Unit, Royal College of Surgeons of England, London, UK; ${ }^{3}$ Southmead Hospital, North Bristol NHS Trust, Bristol, UK; ${ }^{4}$ St. George's Vascular Institute, St. George's Vascular Institute, St. George's Healthcare NHS Trust, London, UK
\end{abstract}

\subsection{6/jech-2017-SSMAbstracts. 137}

Background Availability and diversity of lower limb revascularisation procedures have increased in in the past decade, coinciding with the reconfiguration of vascular services in the United Kingdom. The aim of our study was to investigate whether these developments in care have translated to improvements in patient outcomes.

Methods We used data from Hospital Episode Statistics (HES) to identify patients who underwent endovascular or surgical (endarterectomy, profundaplasty or bypass) lower limb revascularisation for infrainguinal peripheral arterial disease (PAD) in England in 2006-2013. Major lower limb amputations and deaths were ascertained from HES and Office for National Statistics mortality register. Associations of revascularisation procedures with amputation and death outcomes were investigated using Fine-Grey competing risks regression, with adjustment for patient age, sex and comorbidity score. We examined the possible impact of patient selection by stratifying our analyses by indication for revascularisation (intermittent claudication only; severe limb ischaemia without tissue loss; severe limb ischaemia with ulceration; severe limb ischaemia with gangrene).

Results Over the 8 year study period the overall number of endovascular revascularisations increased and the number of surgical procedures decreased: the evidence for this trend was the clearest among patients with the most severe underlying disease (severe limb ischaemia with ulceration or gangrene). The 1 year risk of major amputation reduced from $5.9 \%$ (in 2006-07) to 5.5\% (in 2012-13) following endovascular evascularization and from $10.8 \%(2006-07)$ to $7.4 \%$ (2012-13) following surgical procedures $(\mathrm{p}<0.0001)$. The risk of death after both types of procedures also decreased, whilst the number of comorbidities and the proportions of patients with more severe underlying disease increased.

Discussion Our findings suggest that patient outcomes following lower limb revascularisation have improved during a period of centralisation and specialisation of vascular services in the United Kingdom, despite higher morbidity and an increasing proportion of patients treated for the severe end of the PAD spectrum.

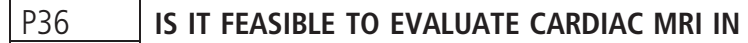 PATIENTS WHO ACTIVATE THE PRIMARY PERCUTANEOUS CORONARY INTERVENTION PATHWAY USING HOSPITAL EPISODE STATISTICS DATA?}

${ }^{1} \mathrm{M}$ Pufulete, ${ }^{1} \mathrm{~J}$ Harris, ${ }^{2} \mathrm{~S}$ Dorman, ${ }^{1} \mathrm{R}$ Brierley*, ${ }^{1} \mathrm{~B}$ Reeves. ${ }^{1}$ Clinical Trials and Evaluation Unit, University of Bristol, Bristol, UK; ${ }^{2}$ Bristol Heart Institute, University Hospitals Bristol NHS Foundation Trust, Bristol, UK

\subsection{6/jech-2017-SSMAbstracts. 138}

Background Cardiac magnetic resonance (CMR) imaging is a non-invasive test used to assess the structure and function of the heart. We tested the feasibility of assembling a retrospective cohort study of patients who activate the primary percutaneous coronary intervention (PPCI) pathway using data from Hospital Episode Statistics (HES) and Patient Episode Database Wales (PEDW) to: i) document the use of cardiac magnetic resonance (CMR) imaging in this population; and ii) determine whether $\mathrm{CMR}$ is associated with improved clinical outcomes.

Methods Patients from four UK sites were recruited into the prospective cohort study. We assembled a database by linking routinely collected hospital data for the index PPCI admission (demography, clinical, biochemistry and imaging) with HES and PEDW describing inpatient and outpatient NHS episodes in the 12 months following the index PPCI admission. We determined whether we could identify the following from HES/PEDW data: i) the index event (cohort entry); ii) CMR within 10 weeks of the index event (exposure); iii) relevant subgroups of the population (e.g. PPCI, unobstructed coronary arteries, multivessel disease, cardiac arrest, etc.); and iv) clinical outcomes.

Results A total of 1670 patients were recruited prospectively into the cohort study; of these 1612 (97\%) had admission data in HES/PEDW that coincided with the index event ( \pm 1 day). Only $1227 / 1612$ (76\%) had HES/PEDW data that met the criteria for cohort entry; 1110 (91\%) were identified as PPCI and 117 (9\%) were identified as emergency angiography only. The remainder $(385 / 1612,24 \%)$ did not meet inclusion criteria (PCI procedure code and ST-elevation myocardial 